\title{
Distributed relative localization using the multi-dimensional weighted centroid
}

\author{
Rosario Aragues ${ }^{1, *}$, Antonio González ${ }^{1}$, Gonzalo López-Nicolás ${ }^{1}$ and Carlos Sagues ${ }^{1}$
}

\begin{abstract}
A key problem in multi-agent systems is the distributed estimation of the localization of agents in a common reference from relative measurements. Estimations can be referred to an anchor node or, as we do here, referred to the weighted centroid of the multi-agent system. We propose a Jacobi OverRelaxation method for distributed estimation of the weighted centroid of the multi-agent system from noisy relative measurements. Contrary to previous approaches, we consider relative multidimensional measurements with general covariance matrices not necessarily diagonal. We prove our weighted centroid method converges faster than anchor-based solutions. We also analyze the method convergence and provide mathematical constraints that ensure avoiding ringing phenomena.
\end{abstract}

Index Terms-Distributed Sensor Networks; Noisy Relative Measurements; Multi-agent Localization; Jacobi OverRelaxation; Weighted Centroid

\section{INTRODUCTION}

Localization is a central task in multi-agent systems. For example, in order to cooperatively manipulate a load, agents need to know their positions in a common frame [1]. Agents usually start at unknown locations, and they can only perceive nearby agents (neighbors). Each agent combines bearing and range measurements [2] of the position of its neighbors and it builds a $2 \mathrm{D}$ or $3 \mathrm{D}$ representation of the relative positions of the neighbors in its own local frame (multi-dimensional relative full-position measurements). This multi-dimensional sensed data is corrupted with noise, whose associated covariance matrices are not necessarily diagonal. In this paper we address the distributed relative localization problem [3]-[14], which consists of combining the noisy relative measurements taken by stationary agents to build an estimate of the agents' positions in a common frame.

\section{A. Related Work on Distributed Relative Localization}

First, we discuss the works from the literature [3]-[14] that address a problem close to the one considered in this paper: a distributed localization scenario with stationary agents that take a single set of measurements of nearby agents. The relative measurements represent the $p$-dimensional fullposition of the neighbors, e.g., 2D or 3D relative positions, being these measurements corrupted with noise. Solutions to this problem often rely on linear methods [15], such as

*Supported by group DGA_T45-17R and projects COMMANDIA SOE2/P1/F0638 (Interreg Sudoe Programme, ERDF), PGC2018-098719-BI00 (MCIU/ AEI/ FEDER, UE), JIUZ-2017-TEC-01 Universidad de Zaragoza, and Fundacion Universitaria Antonio Gargallo (Project 2018/B004)

$*$ Corresponding author. ${ }^{1} \mathrm{R}$. Aragues raragues@unizar.es, A. González angonsor@gmail.com, G. López-Nicolás gonlopez@unizar.es and C. Sagues csagues@unizar.es are with Universidad de Zaragoza and Instituto de Investigación en Ingeniería de Aragón I3A, Spain the Jacobi [3]-[6], the Jacobi Over-Relaxation (JOR) [16], the Successive Over-Relaxation (SOR) [17], [18], the GaussSeidel (GS), and the Richardson's method or gradient-descent strategies [7]-[9], [11]-[14], which are also connected with distributed consensus ideas $[7]-[14]$ and are thus resilient to delays and link failures [3], [7].

In addition to the linear method used, the solutions can be classified according to several ideas that we discuss next. Several works fix an anchor node [3]-[6]. The intuition behind anchors is that it is well known that the relative localization problem can only be solved up to an additive constant [3][13]. This ambiguity can thus be removed by fixing the position of one of the agents (the anchor) at the origin of the common reference frame, while the other agents compute their positions relative to the anchor [3], [6]. The placement of the anchor influences the accuracy of the final results and it is common to analyze the estimation errors at the agents as a function of their distances to the anchor [19]. However, it is also common to assume that the first agent is the anchor. Thus, other works prefer not fixing any anchor [7], [8], [10]-[12], [14] and compute the agents positions relative to a different coordinate frame, for instance, the centroid. Here, we propose using the weighted centroid of the multi-agent system as the origin of the the reference frame, and we do not use any anchor since, as we show, this slows down the process.

Localization methods can also be classified depending on their requirements on additional synchronization strategies, or on the required knowledge of global data for their adjustment. The inconvenience of SOR [17], [18] and GS compared to JOR, Jacobi, Richardson's or gradient-descent methods is that they force a specific state update ordering [15], and thus, they require more sophisticated network synchronization policies. Thus, it is interesting to use methods that do not impose this requirement such as the JOR, Jacobi, Richardson's or gradient-descent methods. Solutions based on the Jacobi [3][6] require fixing an anchor, but a benefit of these solutions is that they do not require additional information for adjusting the algorithm. On the other hand, the Richardson's and gradientdescent methods include a parameter $h$ which must be adjusted using global information on the network topology and on the noise in order to ensure convergence [7]-[9], [11]-[13], which is a limitation. Instead, some versions based on the JOR [16] or on gradient-descent [10] establish values for the parameter $h$ without knowing any global information, e.g., $0<h \leq 1$, which is a strong advantage. This is the approach we follow in this paper.

We can further classify the localization methods depending on whether they consider measurements and states that are scalar values or multi-dimensional variables. Most of the works on distributed localization assume scalar states, or 
diagonal covariance matrices [3], [5], [7]-[13]. In this paper, we consider more realistic scenarios, where the covariance matrices associated to multi-dimensional relative measurements are full (instead of diagonal), since each relative measurement may be the result of fusing different sensory data [2]. An example of these covariance matrices can be seen in Fig. 22 a) (gray ellipses). Dismissing the off-diagonal covariance terms has the effect of missing information, e.g., the line of sight between the agents involved in the relative measurement (Fig. 2[d)). As far as we know, only [4], [6] have addressed multidimensional measurements with full covariance matrices, although in both cases they involved the use of an anchor agent and the Jacobi method in some part of the process.

Finally, most of the previous works on distributed localization discuss the asymptotic convergence of the algorithms, but they do not pay attention to the way in which the solution is achieved. The transient performance is key. In [14], it is studied to establish conditions on the stopping criteria for the relative localization methods. Here, we consider the phenomena of ringing, which appears in some cases in discretetime systems [20]. This makes the estimates at each time step change drastically, making it hard to use these oscillating estimates within a higher level task. Here, we propose using a Jacobi Over-Relaxation (JOR) scheme, that includes a tuning parameter $h$. We also propose mathematical constraints on $h$ to avoid ringing which, remarkably, do not depend on any global information on the network topology or on the noise.

\section{B. Additional Works Related to the Localization Problem}

For the sake of completeness, in this section we give an overview of relevant works connected with localization ideas.

Cooperative localization methods [22], estimate the location of agents that move, so that their relative positions change along time. These methods require the existence of a good quality initial estimate of the localization of the agents in their initial positions, which could be generated by the proposed the method.

The problem of localization in sensor networks [26] is sometimes addressed by placing a small subset of anchor nodes, with known positions in global coordinates. The remaining nodes compute their positions using a weighted average (weighted centroid) of the positions of the other nodes. Here, instead, we do not require any anchor.

Instead of considering noisy measurements of the relative $p$-dimensional full-position of their neighbors, several localization algorithms rely on range-only [23], [24], or bearingonly [25] relative measurements. Other related works involving localization [27] and multi-robot graph-SLAM [17], [18] methods, consider not only the agents' positions, but also their orientations, i.e., they consider full poses. The method presented in this paper can also be applied to scenarios that consider robot poses (orientations and positions), provided the agents perform a synchronization [16], [28] to align their orientations, or they estimate a common orientation for their reference frames in a first stage [29]. In addition, agents can express relative measurements in a common alignment frame using sensors that measure the north (compasses or magnetometers) [30].
Finally, formation control [16] and localization are related problems. Although some works discuss the effect of noise in the final result [16], formation algorithms usually assume noise-free measurements.

\section{Statement of Contributions}

From the previous discussion, we summarize the main ideas and contributions of this paper: $(i)$ We propose a relative distributed localization method that considers noisy multidimensional relative measurements with covariance matrices not necessarily diagonal. (ii) We propose a JOR scheme for connected undirected graphs and establish mathematical constraints on the JOR parameter $h$ to ensure convergence and to avoid ringing. The conditions on $h$ do not depend on any global information on the network topology or on the noise. (iii) We do not fix any anchor. Here, agents compute their locations relative to a common reference frame that depends on the weighted centroid of their initial unknown estimates. We prove this weighted centroid method converges faster than the anchor-based alternative.

Compared to our previous work [4], we avoid using any anchors. In order to avoid rinnging, instead of the Jacobi method used in [4], here we propose a JOR scheme with a tuning parameter $h$.

To reach these goals (no anchor, full covariance matrices, JOR scheme), we cope here with system matrices (Section IV. eq. (12) which do not satisfy the properties (row-stochastic, non-negative, primitive, a single eigenvalue equal to one) used to study the convergence in classical scalar consensus [16], [31]. Thus, we adapt in a non trivial way several results that were established for classical scalar consensus.

\section{Preliminaries}

The ideas that appear in this section are a compilation of similar formulations in [3]-[14], conveniently expressed in terms of multi-dimensional relative measurements.

We let $\mathbf{I}_{n}$ be the $n \times n$ identity matrix, $\mathbf{0}_{n_{1} \times n_{2}}$ be a $n_{1} \times n_{2}$ matrix with all entries equal to 0 , and $\mathbf{1}_{n}$ and $\mathbf{0}_{n}$ be column vectors with its $n$ entries equal to 1 and to 0 . The dimensions are omitted when they can be easily inferred. The Kronecker product is denoted by $\otimes$.

Consider $n \in \mathbb{N}$ stationary agents. Each agent $i \in$ $\{1, \ldots, n\}$ has a $p$-dimensional state $\mathbf{x}_{i} \in \mathbb{R}^{p}$ and it observes the states of a subset of the agents relative to its own state. There are $m$ relative measurements. This information is represented by an undirected graph $\mathcal{G}=(\mathcal{V}, \mathcal{E})$, where $\mathcal{V}=\{1, \ldots, n\}$ are the agents. There is an edge $e=(i, j) \in \mathcal{E}$ from $i$ to $j$ if node $i$ has a relative measurement $\mathbf{z}_{e} \in \mathbb{R}^{p}$ of the state of agent $j$,

$$
\mathbf{z}_{e}=\mathbf{x}_{j}-\mathbf{x}_{i}+\mathbf{v}_{e}, \quad \mathbf{v}_{e} \sim \mathcal{N}\left(\mathbf{0}_{p \times p}, \Sigma_{\mathbf{z}_{e}}\right),
$$

where $\mathbf{v}_{e}$ is a Gaussian additive noise.

Each agent $i$ communicates with its neighbors in the graph $\mathcal{G}$, and we assume $\mathcal{G}$ is connected. A measurement $\mathbf{z}_{e}$ from $i$ to $j$ as in (1) also represents a measurement from $j$ to $i$ by reversing its sign, i.e., $-\mathbf{z}_{e}$. Thus, we define the incidence 
matrix $\mathcal{A} \in\{0,1,-1\}^{n \times m}$ of $\mathcal{G}$ by choosing an orientation for each edge (a source and a destination):

$$
\mathcal{A}_{i, e}=\left\{\begin{array}{ll}
1, & \text { if } e=(j, i) \in \mathcal{E} \\
-1, & \text { if } e=(i, j) \in \mathcal{E} \\
0, & \text { otherwise }
\end{array}, \forall i \in \mathcal{V}, e \in \mathcal{E},\right.
$$

where the edge orientation is encoded by the out-edges ( $e=$ $(i, j) \in \mathcal{E})$ and in-edges $(e=(j, i) \in \mathcal{E})$ of agent $i \in \mathcal{V}$.

We let $\mathbf{z}, \mathbf{v} \in \mathbb{R}^{m p}$ and $\Sigma_{\mathbf{z}} \in \mathbb{R}^{m p \times m p}$ contain the information of all the measurements $\mathbf{z}_{e}, \mathbf{v}_{e}, \Sigma_{\mathbf{z}_{e}}$, following some order $\{1, \ldots, m\}$, i.e.,

$$
\begin{aligned}
\mathbf{z} & =\left(\mathbf{z}_{1}^{T}, \ldots, \mathbf{z}_{m}^{T}\right)^{T}, \quad \mathbf{v}=\left(\mathbf{v}_{1}^{T}, \ldots, \mathbf{v}_{m}^{T}\right)^{T}, \\
\Sigma_{\mathbf{z}} & =\operatorname{blkDiag}\left(\Sigma_{\mathbf{z}_{1}}, \ldots, \Sigma_{\mathbf{z}_{m}}\right) .
\end{aligned}
$$

We assume that the measurements are independent since they were acquired individually by the agents, and thus the covariance matrix $\Sigma_{\mathbf{z}}$ is block diagonal. Note that matrix $\Sigma_{\mathbf{z}}$ would be diagonal for fully uncorrelated noises, and $\Sigma_{\mathbf{z}}=\mathbf{I}_{m p}$ for noise-free data. Also, recall that agents are stationary, i.e., $\mathbf{x}_{i} \in \mathbb{R}^{p}$ is constant, and agents compute their states using the measurements $\mathbf{z}$ they collected initially.

The estimation from relative measurements problem consists of estimating the states of the $n$ agents using $\mathbf{z}$. It is well known [3]-[8], [10]-[14] that in relative localization problems, solutions can be determined only up to an additive constant. Usually, one agent $a \in \mathcal{V}$, e.g., the first one, is taken as an anchor with fixed state, e.g., $\hat{\mathbf{x}}_{a}^{a}=\mathbf{0}$, and the states $\hat{\mathbf{x}}_{i}^{a}$ of all the other agents relative to the anchor are computed. We call such approaches anchor-based and we add the superscript $a$ to their associated variables. We let $\mathcal{V}^{a}=\mathcal{V} \backslash\{a\}$ contain the non-anchor nodes, and $\mathcal{A}^{a} \in \mathbb{R}^{(n-1) \times m}$ be as $\mathcal{A}$ in (2) but without the row associated to the anchor. The Best Linear Unbiased Estimator [3] for $\mathbf{x}_{\mathcal{V}^{a}}^{a}$ is

$$
\begin{aligned}
& \hat{\mathbf{x}}_{\mathcal{V}^{a}}^{a}=\Sigma_{\hat{\mathbf{x}}^{a} a} \eta^{a}, \quad \text { where } \quad \Sigma_{\hat{\mathbf{x}}_{\mathcal{V}^{a}}}=\left(\Upsilon^{a}\right)^{-1}, \\
& \eta^{a}=\left(\mathcal{A}^{a} \otimes \mathbf{I}_{p}\right) \Sigma_{\mathbf{z}}^{-1} \mathbf{z}, \Upsilon^{a}=\left(\mathcal{A}^{a} \otimes \mathbf{I}_{p}\right) \Sigma_{\mathbf{z}}^{-1}\left(\mathcal{A}^{a} \otimes \mathbf{I}_{p}\right)^{T} .
\end{aligned}
$$

We let $\hat{\mathbf{x}}_{\mathcal{V}}^{a} \in \mathbb{R}^{n p}$ and $\Sigma_{\hat{\mathbf{x}}_{\mathcal{V}}^{a}} \in \mathbb{R}^{n p \times n p}$ include the anchor state,

$$
\hat{\mathbf{x}}_{\mathcal{V}}^{a}=\left(\mathbf{0},\left(\hat{\mathbf{x}}_{\mathcal{V}^{a}}^{a}\right)^{T}\right)^{T}, \Sigma_{\hat{\mathbf{x}}_{\mathcal{V}}^{a}}=\operatorname{blk} \operatorname{Diag}\left(\mathbf{0}, \Sigma_{\hat{\mathbf{x}}_{\mathcal{V}^{a}}^{a}}\right) \text {. }
$$

Anchor-based methods make agents compute in a distributed way $\hat{\mathbf{x}}_{\mathcal{V}^{a}}^{a}$ as in 3 satisfying

$$
\Upsilon^{a} \hat{\mathbf{x}}_{\mathcal{V}^{a}}^{a}=\eta^{a} \text {. }
$$

More details on eqs. (3) to (5) can be found, e.g., in [3].

The anchor-free expression is similar to (5), but using the original $\mathcal{A} \in \mathbb{R}^{n \times m}$ instead of $\mathcal{A}^{a} \in \mathbb{R}^{(n-1) \times m}$ :

$$
\begin{aligned}
& \Upsilon \mathbf{x}^{\star}=\eta, \text { where } \eta=\left(\mathcal{A} \otimes \mathbf{I}_{p}\right) \Sigma_{\mathbf{z}}^{-1} \mathbf{z} \text {, and } \\
& \Upsilon=\left(\mathcal{A} \otimes \mathbf{I}_{p}\right) \Sigma_{\mathbf{z}}^{-1}\left(\mathcal{A} \otimes \mathbf{I}_{p}\right)^{T}, \text { where } \\
& \Upsilon=\left[\begin{array}{ccc}
\Upsilon_{11} & \ldots & \Upsilon_{1 n} \\
\vdots & \ddots & \vdots \\
\Upsilon_{n 1} & \ldots & \Upsilon_{n n}
\end{array}\right] \text {, where, } \forall i, j \in \mathcal{V}: \\
& \Upsilon_{i i}=\quad \sum_{e=(i, j) \in \mathcal{E} \text { or } e=(j, i) \in \mathcal{E}}^{\Sigma_{\mathbf{z}_{e}}^{-1},} \\
& \Upsilon_{i j}=-\Sigma_{\mathbf{z}_{e}}^{-1}, \text { if } e=(i, j) \in \mathcal{E} \text { or } e=(j, i) \in \mathcal{E}, \\
& \Upsilon_{i j}=\mathbf{0}_{p \times p}, \text { otherwise. }
\end{aligned}
$$

This anchor-free expression (6) is similar to expressions obtained in, e.g., [8] for the scalar case, being here conveniently adapted to the multi-dimensional case.

Following the same convention as in [3], [14], we will refer to $\hat{x}_{\mathcal{V}}^{a}\left(\sqrt{3}\right.$ to (5) ) and to $\mathrm{x}^{\star}$ (6) as, respectively, the anchor-based and anchor-free optimal estimates. The anchorfree expression (6) is more general than (5). As discussed later in Lemma 3.1 the anchor-free optimal estimates $x^{\star}$ include all anchor-based optimal estimates $\hat{\mathbf{x}}_{\mathcal{V}}^{a}$ as in (3)-(5), plus an additive term, which is equivalent to expressing $\hat{\mathbf{x}}_{\mathcal{V}}^{a}$ relative to a different coordinate frame. The goal is that the agents compute in a distributed and fast way a vector $\mathbf{x}^{\star}$ satisfying (6), as explained next.

\section{Weighted Centroid Reference Frame}

In this section, we describe the proposed method and we establish a theoretical framework to address the stability analysis. First, we define the proposed weighted centroid representation of the agents' states.

Definition 3.1 (Weighted Centroid): Given matrix $\Upsilon$ in (6), we define the weighting matrix $\mathbf{w} \in \mathbb{R}^{n p \times p}$ and weighted centroid matrix $M_{\star}^{c} \in \mathbb{R}^{n p \times n p}$, as follows. Note that $\mathbf{w}$ is not a single vector but several.

$$
\begin{aligned}
\mathbf{w} & =D\left(\mathbf{1}_{n} \otimes \mathbf{I}_{p}\right)=\left[\Upsilon_{11}, \Upsilon_{22}, \ldots, \Upsilon_{n n}\right]^{T}, \text { with } \\
D & =\operatorname{blk} \operatorname{Diag}\left(\Upsilon_{11}, \Upsilon_{22}, \ldots, \Upsilon_{n n}\right), \\
M_{\star}^{c} & =\left(\mathbf{1}_{n} \otimes \mathbf{I}_{p}\right)\left(\mathbf{w}^{T}\left(\mathbf{1}_{n} \otimes \mathbf{I}_{p}\right)\right)^{-1} \mathbf{w}^{T},
\end{aligned}
$$

We define the weighted optimal ${ }^{1}$ estimates $\hat{\mathbf{x}}_{\mathcal{V}}^{c}$ as the optimal estimates $\mathrm{x}_{\mathcal{V}}^{a}$ (3) to (5)) and $\mathrm{x}^{\star}$ (6), with the positions expressed relative to their weighted centroid:

$$
\hat{\mathbf{x}}_{\mathcal{V}}^{c}=\Pi \hat{\mathbf{x}}_{\mathcal{V}}^{a} \text {, with } \quad \Pi=\mathbf{I}_{n p}-M_{\star}^{c} .
$$

As the following results show, the weighted centroid representation of the optimal estimates is unique.

Lemma 3.1 (Anchor-free optimal estimates): The vectors $\mathrm{x}^{\star}$ satisfying the equality given in (6), $\Upsilon \mathrm{x}^{\star}=\eta$, are given by:

$$
\mathbf{x}^{\star}=\hat{\mathbf{x}}_{\mathcal{V}}^{a}+\left(\mathbf{1}_{n} \otimes \mathbf{I}_{p}\right) \mathbf{x}_{a}^{\star}
$$

for all possible $\mathbf{x}_{a}^{\star} \in \mathbb{R}^{p}$, with $\hat{\mathbf{x}}_{\mathcal{V}}^{a}$ as in (4).

Proof: See Appendix A.

Lemma 3.2 (Weighted optimal estimates): Given all possible anchor agents: $a, a^{\prime}, \ldots$, and their associated optimal estimates in (3)-(4), $\hat{\mathbf{x}}_{\mathcal{V}}^{a}, \hat{\mathbf{x}}_{\mathcal{V}}^{a^{\prime}}, \ldots$ obtained by the selected anchor: $a, a^{\prime}, \ldots$, the weighted optimal estimates $\hat{\mathbf{x}}_{\mathcal{V}}^{c}, \hat{\mathbf{x}}_{\mathcal{V}}^{c^{\prime}}, \ldots$ obtained with 8 are the same:

$$
\hat{\mathbf{x}}_{\mathcal{V}}^{c}=\Pi \hat{\mathbf{x}}_{\mathcal{V}}^{a}=\hat{\mathbf{x}}_{\mathcal{V}}^{c^{\prime}}=\Pi \hat{\mathbf{x}}_{\mathcal{V}}^{a^{\prime}}
$$

Proof: See Appendix A

Remark 3.1: Figure 3 shows some examples of weighted optimal estimates $\hat{\mathbf{x}}_{\mathcal{V}}^{c}$ (Def. 3.1 and optimal estimates in (3)-4) (red circles). These values can only be computed immediately if the multi-agent system is centralized, e.g., if all relative position measurements are available to each

\footnotetext{
${ }^{1}$ We use the term weighted optimal estimates for $\hat{\mathbf{x}}_{\mathcal{V}}^{c}$ following the same naming convention in [3], [14], as we did for the anchor-based and anchorfree optimal estimates.
} 
agent. Since we deal with distributed systems, we have to compute these values iteratively. In a distributed scenario, each agent $i \in \mathcal{V}$ only knows the relative measurements $\mathbf{z}_{e}, \Sigma_{\mathbf{z}_{e}}$ associated to its neighbors, $e=(j, i) \in \mathcal{E}$ or $e=(i, j) \in \mathcal{E}$, and the estimate of its own position. Besides, each agent $i$ can only exchange its estimated position with its neighbors. The goal is that agents iteratively obtain an estimated position that converges asymptotically to the same optimal estimates or weighted optimal estimates as the ones discussed so far. In the next sections, we present a distributed solution to this problem and we discuss its properties.

\section{Weighted Centroid Localization}

In this section, we present a distributed iterative method to let each agent $i$ estimate its associated entries within $\mathbf{x}^{\star}$ (6). We use a Jacobi Over-Relaxation scheme and discuss the selection of its parameter $h$. From all the possible vectors $\mathbf{x}^{\star}$, we prove that the agents' estimates converge to an expression that depends on the weighted centroid of the initial estimates.

Matrix $\Upsilon$ in (6) is decomposed into a matrix $D$ with the $p \times p$ blocks in the main diagonal of $\Upsilon$, and a matrix $N$ with the remaining elements,

$$
\begin{aligned}
& \Upsilon=D-N, \text { with } D=\operatorname{blkDiag}\left(\Upsilon_{11}, \Upsilon_{22}, \ldots, \Upsilon_{n n}\right), \\
& N=\left[\begin{array}{ccc}
N_{11} & \ldots & N_{1 n} \\
\vdots & \ddots & \vdots \\
N_{n 1} & \cdots & N_{n n}
\end{array}\right] \text {, where, } \forall i, j \in \mathcal{V}: \\
& N_{i j}= \begin{cases}\Sigma_{\mathbf{z}_{e}}^{-1} & \text { if } e=(i, j) \in \mathcal{E} \text { or } e=(j, i) \in \mathcal{E}, \\
0 & \text { otherwise. }\end{cases}
\end{aligned}
$$

From this, the JOR system equations for multi-dimensional noisy centroid-based localization used to compute $x^{\star}$ (6) in a distributed way are:

$$
\begin{aligned}
\mathbf{x}(k+1) & =M_{\mathrm{JOR}} \mathbf{x}(k)+h D^{-1} \eta, \text { with } \\
M_{\mathrm{JOR}} & =(1-h) \mathbf{I}_{n p}+h D^{-1} N=\mathbf{I}_{n p}-h D^{-1} \Upsilon .
\end{aligned}
$$

Matrix $M_{\mathrm{JOR}}$ does not change at each iteration. The method is distributed and each agent $i$ runs (12) to estimate its entries $x_{i}(k) \in \mathbb{R}^{p}$ within $\mathbf{x}(k)$ using local information and from its neighbors:

$$
\begin{aligned}
& x_{i}(k+1)=h \Upsilon_{i i}^{-1}\left(\sum_{e=(i, j) \in \mathcal{E}} \Sigma_{\mathbf{z}_{e}}^{-1}\left(x_{j}(k)-\mathbf{z}_{e}\right)\right) \\
& +h \Upsilon_{i i}^{-1}\left(\sum_{e=(j, i) \in \mathcal{E}} \Sigma_{\mathbf{z}_{e}}^{-1}\left(x_{j}(k)+\mathbf{z}_{e}\right)\right)+(1-h) x_{i}(k) .
\end{aligned}
$$

The multi-dimensional noisy centroid-based distributed localization method $(12)$ is connected with scalar weighted consensus problems [31], [16]. However, $M_{\mathrm{JOR}}$ in (12) does not satisfy the properties (row-stochastic, non-negative, primitive, a single eigenvalue equal to one) used in classical scalar consensus [31], [16] to establish the convergence for connected graphs. We extend several properties from scalar weighted consensus and we show that algorithm (12) makes the agents states converge to an expression that depends on the weighted centroid (Def. 3.1). Lemmas 4.1 and 4.2 study the solutions of the JOR equations, and the eigenvectors of matrix $M_{\mathrm{JOR}}$, and Propositions 4.1 and 4.2 study the convergence of the resulting closed-loop system. These results are used to prove Theorem 4.1. The proofs of these results appear at Appendices $\mathrm{B}$ to $\mathrm{E}$, and use the following definitions.

Definition 4.1 (Anchor-based JOR matrix): We let $M_{\mathrm{JOR}}^{a}$, $\Upsilon^{a}, D^{a}, N^{a}$ be as $M_{\mathrm{JOR}}, \Upsilon, D, N$ in $(11)-12$ but removing the rows and columns associated to the anchor agent.

Definition 4.2 (Spectral radius and convergence rates): We let $\rho(M)$ be the spectral radius of a matrix $M$. We define the convergence rate of the anchor-based method as $\rho\left(M_{\mathrm{JOR}}^{a}\right)$, and the convergence rate of the weighted centroid algorithm as $\rho_{\text {ess }}\left(M_{\mathrm{JOR}}\right)=\rho\left(M_{\mathrm{JOR}}-M_{\star}^{c}\right)$, with $M_{\mathrm{JOR}}^{a}, M_{\star}^{c}, M_{\mathrm{JOR}}$ as in Def. 4.1, and in (7), (12).

Definition 4.3 (Eigenvalues of JOR and Jacobi matrices): Let $M_{\mathrm{JOR}}, \Upsilon, D, N$ be as in $(11)-(12)$, and $M_{\mathrm{JOR}}^{a}$, $\Upsilon^{a}, D^{a}, N^{a}$ be as in Def. 4.1. The Jacobi matrices associated to the JOR matrices $M_{\mathrm{JOR}}$ and $M_{\mathrm{JOR}}^{a}$ are, respectively, $D^{-1} N$ and $\left(D^{a}\right)^{-1} N^{a}$. We use the following notation to refer to the eigenvalues of the JOR and Jacobi matrices:

$$
\begin{aligned}
& \lambda_{i, r}\left(M_{\mathrm{JOR}}\right), \lambda_{i, r}\left(D^{-1} N\right), \text { for } i=1, \ldots, n, r=1, \ldots, p . \\
& \lambda_{i, r}^{a}\left(M_{\mathrm{JOR}}^{a}\right), \lambda_{i, r}^{a}\left(\left(D^{a}\right)^{-1} N^{a}\right), \\
& \quad \text { for } i=1, \ldots, n-1, r=1, \ldots, p .
\end{aligned}
$$

Lemma 4.1 (Solutions of the JOR equations): The vectors $\mathrm{x}^{\star}$ satisfying (11)-12, i.e.,

$$
\mathbf{x}^{\star}=M_{\mathrm{JOR}} \mathbf{x}^{\star}+h D^{1} \eta,
$$

where $\eta, D$ and $M_{\mathrm{JOR}}$ are defined in (6), 11) and (12), are given by

$$
\mathbf{x}^{\star}=\hat{\mathbf{x}}_{\mathcal{V}}^{a}+\left(\mathbf{1}_{n} \otimes \mathbf{I}_{p}\right) \mathbf{x}_{a}^{\star}
$$

for all possible $\mathbf{x}_{a}^{\star} \in \mathbb{R}^{p}$, with $\hat{\mathbf{x}}_{\mathcal{V}}^{a}$ as in (4).

Proof: See Appendix B.

Lemma 4.2 (Eigenvectors of the JOR matrix): The weighting matrix in Definition 3.1 are left eigenvectors of the $M_{\mathrm{JOR}}$ system matrix (12), associated to the eigenvalue 1 ,

$$
\mathbf{w}^{T} M_{\mathrm{JOR}}=\mathbf{w}^{T} .
$$

In addition, the following results hold:

$$
\begin{aligned}
\Upsilon \cdot\left(\mathbf{1}_{n} \otimes \mathbf{I}_{p}\right) & =\mathbf{0}_{n p \times p}, \text { and } \\
M_{\mathrm{JOR}} \cdot\left(\mathbf{1}_{n} \otimes \mathbf{I}_{p}\right) & =\mathbf{1}_{n} \otimes \mathbf{I}_{p},
\end{aligned}
$$

with $\Upsilon$ as in 6 .

Proof: See Appendix B

Proposition 4.1 (Eigenvalues of JOR): For connected graphs $\mathcal{G}$, with $0<h<1$, the JOR system matrix $M_{\mathrm{JOR}}$ in (12) has $p$ eigenvalues equal to 1 , and the remaining eigenvalues are real and have modulus strictly smaller than one.

Proof: See Appendix C

Proposition 4.2 (Convergence of the powers of the JOR matrix): Let $M_{\mathrm{JOR}}$ be the system matrix associated to a connected graph $\mathcal{G}$ and the JOR iterations as described in (12). Let $M_{\star}^{c}$ be as in Definition 3.1 and let $0<h<1$. Then,

$$
\lim _{k \rightarrow \infty}\left(M_{\mathrm{JOR}}\right)^{k}=M_{\star}^{c} \text {. }
$$



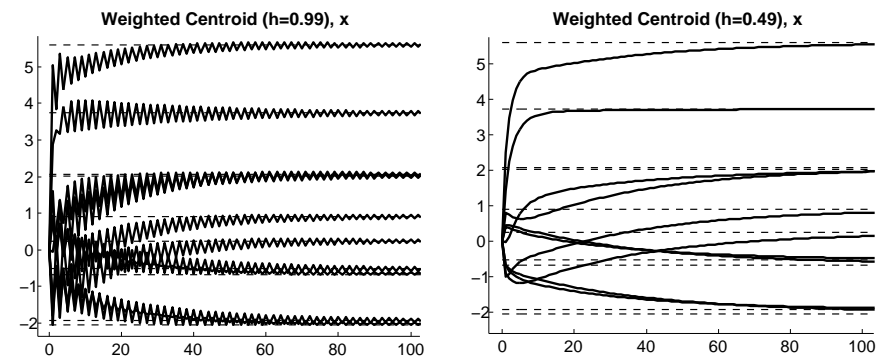

Fig. 1. Example with 10 agents in a chain graph. Evolution along iterations of the estimated $x$-coordinate relative to the weighted centroid of the team. Left: The ringing oscillatory behavior can be observed for $h=0.99$. At each step, the estimates change their values sharply. Right: The ringing oscillatory behavior is removed with $h=0.49$. The estimates converge now smoothly.

Proof: See Appendix D

Now we present the main result in this section.

Theorem 4.1 (JOR Weighted Centroid iterations): If agents execute the multi-dimensional noisy centroid-based localization method (11)-(12) under a connected graph $\mathcal{G}$, with $0<h<1$, their estimates asymptotically converge to the optimal estimates relative to the centroid $\hat{\mathbf{x}}_{\mathcal{V}}^{c}$ (Definition 3.1, plus the weighted centroid of the initial states,

$$
\lim _{k \rightarrow \infty} \mathbf{x}(k)=\hat{\mathbf{x}}_{\mathcal{V}}^{c}+M_{\star}^{c} \mathbf{x}(0) .
$$

Proof: See Appendix E,

\section{CONDITIONS ON $h$ FOR AVOIDING Ringing}

The convergence of the JOR localization method is asymptotic (Theorem 4.1). In the literature on relative localization, it is common to focus on studying the asymptotic convergence of the methods. Aside from mere stability analysis, the transient performance is a key aspect to take into account [14]. Not only convergence is a necessary requirement in practice, but it is also convenient that localization estimates evolve smoothly without oscillating behaviors. This would allow using stable and predictable data in higher level methods. It is well known that real and positive eigenvalues implies the absence of oscillatory behavior (ringing) [20]. Conversely, if poles are negative or have imaginary components, undesirable oscillations will appear. An example of a convergent system with this behavior can be seen in Fig. 1. Thus, we propose alleviating this behavior by forcing the system eigenvalues to be real, positive, and smaller than 1 , as we propose next.

Lemma 5.1: The system matrix $M_{\mathrm{JOR}}$ in 12 with $0<h<$ $1 / 2$ and $\mathcal{G}$ connected, has $p$ eigenvalues equal to 1 , and all its remaining eigenvalues are real, strictly positive, and smaller than 1.

Proof: See Appendix F,

Observe in Fig. 1 this ringing behavior is avoided with $h$ close to $1 / 2$. Note that the selection of $0<h<1 / 2$ does not require knowing global information of the graph.

In our previous work [4], we used the Jacobi algorithm, which is like the JOR with $h=1$. This condition does not satisfy Lemma 5.1, and therefore, the method given in [4] and other Jacobi-based approaches, cannot guarantee the removal of the ringing behavior.
Observe that the interest of the conditions on $h$ given in Theorem 4.1 and Lemma 5.1 is that they only require $\mathcal{G}$ to be connected. Additional information could be used to speed up the method as follows. Suppose that we knew the eigenvalues of the Jacobi matrix $D^{-1} \Upsilon$ (Def. 4.3), and let $\lambda_{\max }\left(D^{-1} \Upsilon\right)$ and $\lambda_{\min }\left(D^{-1} \Upsilon\right)$ be respectively its maximum and its minimum nonzero eigenvalues. Observe that matrices $D^{-1} \Upsilon$ and $M_{\mathrm{JOR}}$ are related by $M_{\mathrm{JOR}}=\mathbf{I}_{n p}-h D^{-1} \Upsilon$ (eq. (12), and thus, their eigenvalues (Def. 4.3 are related by $\lambda_{i, r}\left(M_{\mathrm{JOR}}\right)=1-h \lambda_{i, r}\left(D^{-1} \Upsilon\right)$, for all $i \in \mathcal{V}, r=1, \ldots, p$. Thus, we could follow a similar reasoning as it is often done for relating Perron and Laplacian matrices, e.g., as in [32]. We could easily build optimal expressions on $h$ from the point of view of the speed of convergence. For instance, if we want the eigenvalues of $M_{\mathrm{JOR}}$ to remain positive while speeding up the method, we could use $h=\frac{1}{\lambda_{\max }\left(D^{-1} \Upsilon\right)}$. If instead, we want to speed up the method, allowing for both positive and negative eigenvalues in $M_{\mathrm{JOR}}$, we could use $h=\frac{2}{\lambda_{\min }\left(D^{-1} \Upsilon\right)+\lambda_{\max }\left(D^{-1} \Upsilon\right)}$. While these adjustments of $h$ will give rise to a faster convergence, the main inconvenience compared to the approach proposed in this paper, is that they require prior knowledge of the eigenvalues of matrix $D^{-1} \Upsilon$, which has dependence on the graph structure and the noise covariance matrices of measured data. Thus, we prefer for generality to use Lemma 5.1 and avoid requirements on global data.

\section{COMPARISON BETWEEN ANCHOR AND CEnTROID-BASED STRATEGIES}

Both anchor-based and weighted centroid localization methods are convergent for connected graphs. However, we demonstrate next that our proposed weighted centroid approach converges faster than fixing an anchor node.

Lemma 6.1: Let $0<h<1 / 2$ and $\mathcal{G}$ be connected. The convergence rate of the anchor-based $\rho\left(M_{\mathrm{JOR}}^{a}\right)$ and of the weighted centroid algorithms $\rho_{\text {ess }}\left(M_{\mathrm{JOR}}\right)=\rho\left(M_{\mathrm{JOR}}-M_{\star}^{c}\right)$ (Definition 4.2), satisfy

$$
\rho_{\text {ess }}\left(M_{\mathrm{JOR}}\right) \leq \rho\left(M_{\mathrm{JOR}}^{a}\right) .
$$

Proof: See Appendix F.

\section{Simulations}

Fig. 2 illustrates the difference of performance discussed in Lemma 6.1 There are 10 agents placed randomly in a 2D region of $10 \times 10$ meters. Fig. 2 (a) Each agent $i$ gets noisy measurements (crosses and ellipses) of the relative position (arrows) of some of its neighbors $j$ (out-edges $e=(i, j) \in$ $\mathcal{E})$. Equivalently, all agents in the neighborhood of agent $i$ know the noisy measurements of the relative position of agent $i$. The noise covariance matrix $\Sigma_{\mathbf{z}_{e}}$ depends on the relative measurements $\left(\rho_{i j}, \alpha_{i j}\right.$ in polar coordinates) between agents $i, j$, with $e=(i, j)$ as follows:

$$
\Sigma_{\mathbf{z}_{e}}=R_{i j}^{T} \operatorname{diag}\left(\sigma_{1}^{2}, \sigma_{2}^{2}\right) R_{i j},
$$

where $\sigma_{1}=0.15 \rho_{i j}, \sigma_{2}=0.1 \rho_{i j}$, are the standard deviations in the parallel and perpendicular directions of the arrow, and

$$
R_{i j}=\left[\begin{array}{cc}
\cos \left(\alpha_{i j}\right) & \sin \left(\alpha_{i j}\right) \\
-\sin \left(\alpha_{i j}\right) & \cos \left(\alpha_{i j}\right)
\end{array}\right] \text {. }
$$




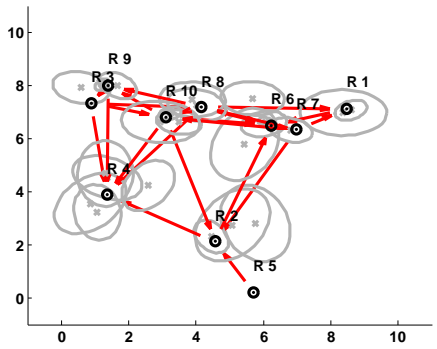

(a)

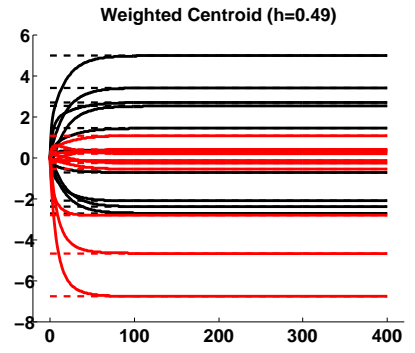

(b)

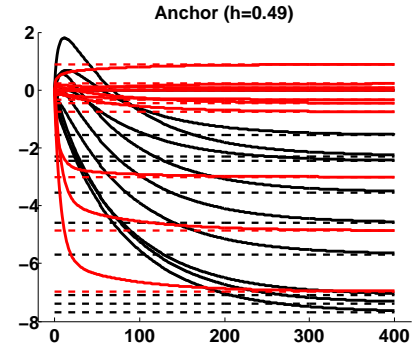

(c)

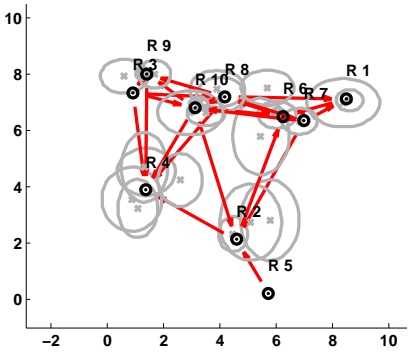

(d)

Fig. 2. (a): Initial scenario. (b), (c): Evolution of the estimated $x-$ (in black) and $y$ - coordinates (in red) of the agents positions along 400 iterations ( $x-$ axis) when agents run the weighted centroid (b) and anchor-based (c) localization methods. (d): The same example as in (a), but with relative measurements with diagonal covariances to show that, contrary to our proposal, the directionality of the information is lost.
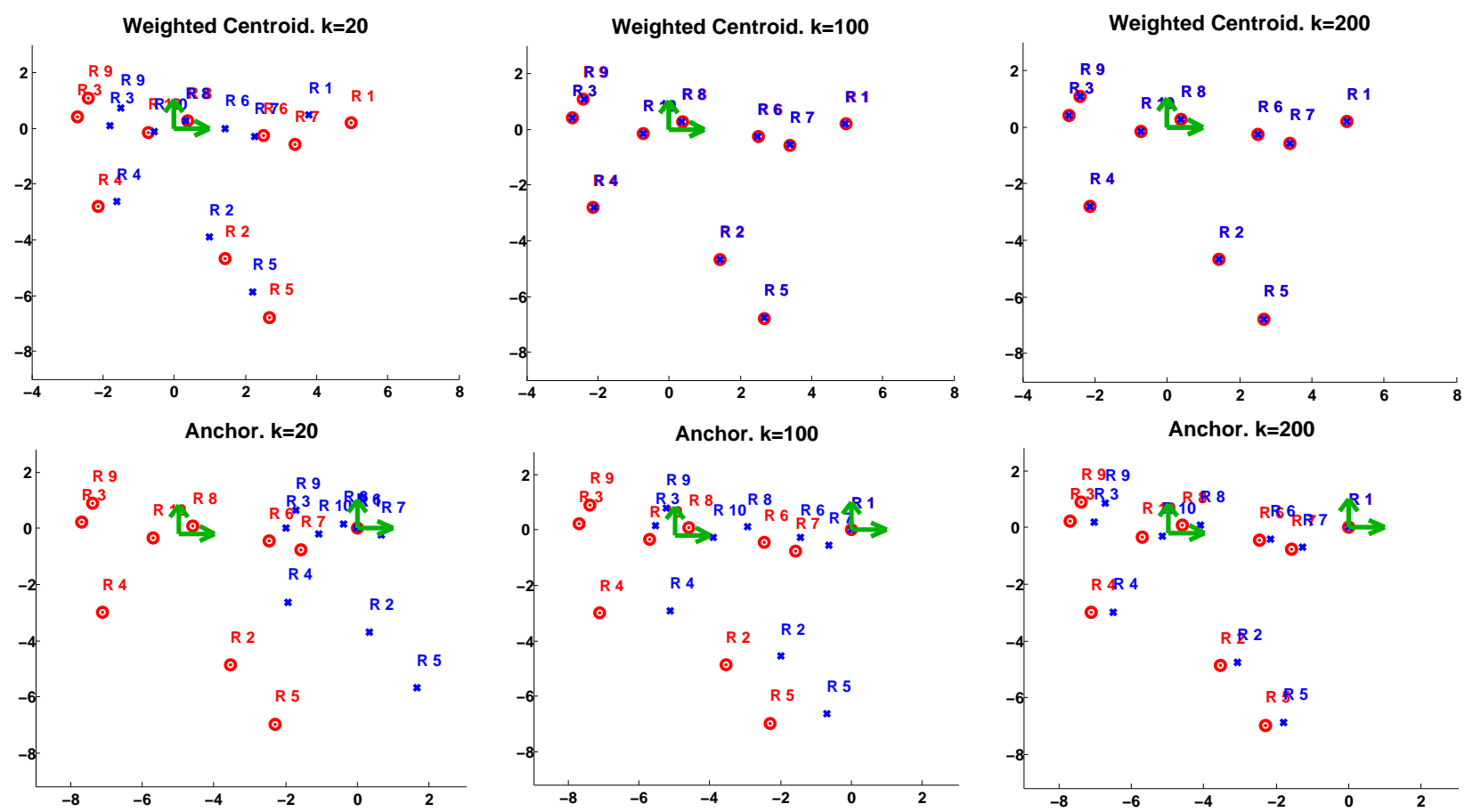

Fig. 3. Estimated agents positions (blue crosses) after $k=20$ (Left), $k=100$ (Center) and $k=200$ iterations (Right) of the weighted centroid (Top) and anchor-based (Bottom) localization methods, for the initial scenario in Fig. 2 The red circles represent the optimal estimates in 3 - 47, with the positions expressed relative to their weighted centroid as in [8) (Top), and relative to the anchor agent $R 1$ (Bottom). The green axis represent the frame used as the origin. In the bottom figures (anchor-based methods), we also display with a green axis the weighted centroid, which is located at position ( $-4.97,-0.19)$.

We plot the uncertainty ellipses with the $95 \%$ of noisy measurements $\left( \pm 2 \sigma_{1}, \pm 2 \sigma_{2}\right)$, centered around the noisy measurement. Circles represent instead the true initial robot positions. Note that the covariance matrices associated to the relative measurements are full, i.e., they are not diagonal. For instance, for the measurement between agents $i=5$ and $j=2$, we have

$$
\Sigma_{\mathbf{z}_{e}}=\left[\begin{array}{cc}
0.0659 & -0.0273 \\
-0.0273 & 0.0954
\end{array}\right] .
$$

This covariance matrix is full, and the uncertainty ellipse is correctly aligned with the direction of the relative measurement between agents $i=5$ and $j=2$. Thus, our method can be applied to relative measurements obtained with sensors such as stereo cameras, or RGB-D devices, that have different precision in the depth and in the orientation data. Note from Fig. 2(d) that, since we consider the off diagonal terms in the covariance matrices, we do not lose any information about the direction of the relative measurements. Fig. 2(b): Agents run the distributed weighted centroid localization method with $h=0.49$ in order to avoid ringing (Lemma 5.1). The estimates converge fast and without exhibiting the ringing oscillatory behavior. Fig. 2(c): The estimated agent positions when fixing node 1 as an anchor at the $(0,0)$ position, which clearly converge slower than using the weighted centroid approach, as stated by Lemma 6.1. Fig. 3 shows the evolution of the estimated agents positions for the methods in Fig. 2. After few steps, the estimated positions (blue crosses) obtained with the distributed weighted centroid localization (Fig. 3, top) are very close to the optimal estimates (red circles). Fig. 3 bottom: after $k=200$ iterations, the estimated agent positions (blue circles) when fixing node 1 as an anchor at the $(0,0)$ position, have not converged yet to the optimal estimates (red circles). 


\section{CONCLUSIONS}

We have presented a distributed method that allows a set of agents to estimate their positions, expressed relative to the weighted centroid, using noisy relative measurements of the positions of their neighbors. Our method is based on the Jacobi Over-Relaxation (JOR), and it includes a tuning parameter $h$. A novel feature of our JOR based distributed method is that the noisy relative measurements can be multidimensional with full covariance matrices, as opposed to the common assumption in the literature of diagonal covariance matrices. Thus, our approach is more flexible and practical, since very often the measurements are fused from several sensors. We also defined the conditions that guarantee smoother performance of the estimates. This is a desirable feature if, for example, the estimates are used in a higher level task sensitive to oscillating signals. Additionally, we proved our weighted-centroid method converges faster than its counterpart based on a fixed anchor.

\section{A: Proofs of Lemmas 3.1 AND 3.2}

Proof of Lemma 3.1. We focus on (6). The relation between the incidence matrices, with and without the anchor row is

$$
\mathcal{A}=\left[\begin{array}{c}
-\mathbf{1}_{n-1}^{T} \\
\mathbf{I}_{n-1}
\end{array}\right] \mathcal{A}^{a}, \quad \mathcal{A}^{a}=\left[\begin{array}{ll}
\mathbf{0}_{n-1} & \mathbf{I}_{n-1}
\end{array}\right] \mathcal{A} .
$$

$\Upsilon \mathrm{x}^{\star}=\eta$ (6) can be expressed, distinguishing between the elements of $\mathbf{x}^{\star}=\left[\mathbf{x}_{a}^{\star},\left(\mathbf{x}_{\mathcal{V}^{a}}^{\star}\right)^{T}\right]^{T}$,

$$
\left(\mathcal{A} \otimes \mathbf{I}_{p}\right) \Sigma_{\mathbf{z}}^{-1}\left(\mathcal{A}^{a} \otimes \mathbf{I}_{p}\right)^{T}\left(\mathbf{x}_{\mathcal{V}^{a}}^{\star}-\left(\mathbf{1}_{n-1} \otimes \mathbf{I}_{p}\right) \mathbf{x}_{a}^{\star}\right)=\eta,
$$

giving two rows of equations. The first row is redundant (it equals the second row, multiplied by $-\mathbf{1}_{n-1}^{T} \otimes \mathbf{I}_{p}$ ). Using $\Upsilon^{a}$, $\eta^{a}$ (3), the second row is:

$$
\Upsilon^{a}\left(\mathbf{x}_{\mathcal{V}^{a}}^{\star}-\left(\mathbf{1}_{n-1} \otimes \mathbf{I}_{p}\right) \mathbf{x}_{a}^{\star}\right)=\eta^{a}
$$

Since $\Upsilon^{a} \hat{\mathbf{x}}_{\mathcal{V}^{a}}^{a}=\eta^{a}(5)$, then all $\mathbf{x}^{\star}$ satisfy

$$
\hat{\mathbf{x}}_{\mathcal{V}^{a}}^{a}=\mathbf{x}_{\mathcal{V}^{a}}^{\star}-\left(\mathbf{1}_{n-1} \otimes \mathbf{I}_{p}\right) \mathbf{x}_{a}^{\star}
$$

and, finally, $\hat{\mathbf{x}}_{\mathcal{V}}^{a}=\mathbf{x}^{\star}-\left(\mathbf{1}_{n} \otimes \mathbf{I}_{p}\right) \mathbf{x}_{a}^{\star}$ as in (9).

Proof of Lemma 3.2. From Lemma 3.1. vectors $\mathbf{x}^{\star}$ satisfying (6) include all anchor-based vectors $\hat{\mathbf{x}}_{\mathcal{V}}^{a}$ as in (3) (5), plus an additive term, which is equivalent to expressing $\hat{\mathbf{x}}_{\mathcal{V}}^{a}$ relative to a different coordinate frame, so that

$$
\hat{\mathbf{x}}_{\mathcal{V}}^{a}=\hat{\mathbf{x}}_{\mathcal{V}}^{a^{\prime}}+\left(\mathbf{1}_{n} \otimes \mathbf{I}_{p}\right) \hat{\mathbf{x}}_{a^{\prime}}^{a}
$$

Substituting this in $(8)$, we get

$$
\begin{aligned}
\hat{\mathbf{x}}_{\mathcal{V}}^{c} & =\Pi \hat{\mathbf{x}}_{\mathcal{V}}^{a}=\Pi\left(\hat{\mathbf{x}}_{\mathcal{V}}^{a^{\prime}}+\left(\mathbf{1}_{n} \otimes \mathbf{I}_{p}\right) \hat{\mathbf{x}}_{a^{\prime}}^{a}\right), \\
& =\Pi \hat{\mathbf{x}}_{\mathcal{V}}^{a^{\prime}}+\left(\mathbf{I}_{n p}-M_{\star}^{c}\right)\left(\mathbf{1}_{n} \otimes \mathbf{I}_{p}\right) \hat{\mathbf{x}}_{a^{\prime}}^{a} .
\end{aligned}
$$

Now we use (7), that gives $M_{\star}^{c}\left(\mathbf{1}_{n} \otimes \mathbf{I}_{p}\right)=\left(\mathbf{1}_{n} \otimes \mathbf{I}_{p}\right)$, which combined with (25), gives $\hat{\mathbf{x}}_{\mathcal{V}}^{c}=\Pi \hat{\mathbf{x}}_{\mathcal{V}}^{a^{\prime}}+\mathbf{0}=\hat{\mathbf{x}}_{\mathcal{V}}^{c^{\prime}}$ as in (10), concluding the proof.

\section{B: Proofs of Lemmas 4.1 And 4.2}

Proof of Lemma 4.1. Since all $\mathrm{x}^{\star}$ satisfying (14) also satisfy (6), the proof is completed by using Lemma 3.1

Proof of Lemma 4.2. From the definition of $\Upsilon$ in (6), (17) immediately follows. Now we consider $M_{\mathrm{JOR}}$ in (12):

$$
M_{\mathrm{JOR}} \cdot\left(\mathbf{1}_{n} \otimes \mathbf{I}_{p}\right)=\mathbf{1}_{n} \otimes \mathbf{I}_{p}-h \Upsilon \cdot\left(\mathbf{1}_{n} \otimes \mathbf{I}_{p}\right),
$$

where the second term vanishes (17), and we get (18). Finally, from $M_{\mathrm{JOR}}$ in (12), we have

$$
\mathbf{w}^{T} M_{\mathrm{JOR}}=\mathbf{w}^{T}-h \mathbf{w}^{T} D^{-1} \Upsilon
$$

which combined with Def. 3.1 gives

$$
\mathbf{w}^{T} M_{\mathrm{JOR}}=\mathbf{w}^{T}-h\left(\mathbf{1}_{n} \otimes \mathbf{I}_{p}\right)^{T} \Upsilon .
$$

Since $\Upsilon$ is symmetric, the term $\left(\mathbf{1}_{n} \otimes \mathbf{I}_{p}\right)^{T} \Upsilon$ also vanishes in $(12)$, and we finally get $(16)$, concluding the proof.

\section{C: Proof of Proposition 4.1}

In this proof, we compare the eigenvalues of matrices $M_{\mathrm{JOR}}$ (12) and $M_{\mathrm{JOR}}^{a}$ (Def.4.1). To study their properties, we relate $M_{\mathrm{JOR}}$ and $M_{\mathrm{JOR}}^{a}$ with symmetric matrices, and with Jacobi matrices (Def. 4.3), using the following auxiliary results.

Lemma C.1 (Matrix similarity): Matrices $M_{\mathrm{JOR}}$ in (12) and $M_{\mathrm{JOR}}^{a}$ in Definition 4.1 are similar, according to [33. Definition 1.3.1], to the following symmetric matrices:

$$
\begin{aligned}
M_{\mathrm{JOR}} & \backsim D^{1 / 2} M_{\mathrm{JOR}} D^{-1 / 2}=(1-h) \mathbf{I}_{n p}+h D^{-1 / 2} N D^{-1 / 2} . \\
M_{\mathrm{JOR}}^{a} & \backsim\left(D^{a}\right)^{1 / 2} M_{\mathrm{JOR}}^{a}\left(D^{a}\right)^{-1 / 2} \\
& =(1-h) \mathbf{I}_{(n-1) p}+h\left(D^{a}\right)^{-1 / 2} N^{a}\left(D^{a}\right)^{-1 / 2} .
\end{aligned}
$$

$M_{\mathrm{JOR}}$ and $M_{\mathrm{JOR}}^{a}$ have the same eigenvalues as their similar symmetric counterparts ( [33, Corollary 1.3.4]). Matrices $M_{\mathrm{JOR}}$ and $M_{\mathrm{JOR}}^{a}$ are diagonalizable ( [33, Theorem 4.1.5], [33. Observation 1.3.2], [33, Theorem 1.3.7]). The eigenvalues of $M_{\mathrm{JOR}}$ and $M_{\mathrm{JOR}}^{a}$ are real ( [33, Theorem 4.1.3]).

Proof of Lemma C.1. The fact that matrices $D^{1 / 2} M_{\mathrm{JOR}} D^{-1 / 2}$ and $\left(D^{a}\right)^{1 / 2} M_{\mathrm{JOR}}^{a}\left(D^{a}\right)^{-1 / 2}$ are symmetric is concluded from (29) and from the fact that $N$ (11) and $N^{a}$ (Def.4.1) are symmetric. The remaining ideas follow from using the mentioned results in [33] with symmetric matrices.

Lemma C.2 (Spectral radius of Jacobi matrices): Let $\Upsilon, D$, and $N$ be as in (11), let $D^{a}, N^{a}$ be as in Definition 4.1, and let graph $\mathcal{G}$ be connected. The spectral radii (Def. 4.2) of the following Jacobi matrices satisfy:

$$
\rho\left(D^{-1} N\right) \leq 1, \text { and } \rho\left(\left(D^{a}\right)^{-1} N^{a}\right)<1
$$

Proof of Lemma C.2. We first consider the Jacobi matrix $D^{-1} N$. According to [34, Notation 2.3], which studies the convergence of block iterative methods, matrix $\Upsilon$ in 6 is of type $Z_{n}^{p}$ and also of type $\hat{Z}_{n}^{p}$ for a connected graph. Matrix $\Upsilon$ also satisfies [34, Condition (3.2), Proposition 3.1]:

$$
\Upsilon_{i i}+\frac{1}{2} \sum_{j \neq i, j=1}^{n}\left(\Upsilon_{i j}+\Upsilon_{j i}\right)^{T} \geq 0, \forall i \in \mathcal{V} .
$$


This comes from the fact that, from (6), we have $\Upsilon_{j i}=\Upsilon_{i j}^{T}$ and $\Upsilon_{i j}+\Upsilon_{j i}^{T}=2 \Upsilon_{i j}$, so that 31 gives

$$
\Upsilon_{i i}+\sum_{j \neq i, j=1}^{n} \Upsilon_{i j}=\mathbf{0}_{p \times p} \geq 0, \forall i \in \mathcal{V} .
$$

Therefore, according to [34, Proposition 4.6], $\rho\left(D^{-1} N\right) \leq 1$, with $\Upsilon, D$, and $N$ as in (11).

The convergence of the anchor-based Jacobi method was proved in [4, Theorem 2], using results from [34], and concluding that, for connected graphs, $\rho\left(\left(D^{a}\right)^{-1} N^{a}\right)<1$.

Proof of Proposition 4.1. From Lemma 4.2, we conclude that $M_{\mathrm{JOR}}$ has at least $p$ eigenvalues equal to one. Next, we study if the modulus of the remaining eigenvalues is strictly less than 1 .

We pay attention to $M_{\mathrm{JOR}}$ in (12) and to the Jacobi matrix $D^{-1} N$. From Lemma C.2 $\rho\left(D^{-1} N\right) \leq 1$, with $\Upsilon$, $D$, and $N$ as in (11). The eigenvalues of the JOR system matrix (12) and of the Jacobi matrix $D^{-1} N$ (Def. 4.3 are related $\forall i \in$ $\mathcal{V}, r=1, \ldots, p$ by

$$
\lambda_{i, r}\left(M_{\mathrm{JOR}}\right)=1-h+h \lambda_{i, r}\left(D^{-1} N\right),
$$

Since $h>0$, and using (30), we get:

$$
\begin{aligned}
& -h \leq h \lambda_{i, r}\left(D^{-1} N\right) \leq h, \\
& 1-2 h \leq \lambda_{i, r}\left(M_{\text {JOR }}\right) \leq 1-h+h=1 .
\end{aligned}
$$

Now, using $h<1$, we get that, $\forall i \in \mathcal{V}, r=1, \ldots, p$,

$$
-1<\lambda_{i, r}\left(M_{\mathrm{JOR}}\right) \leq 1 \text {. }
$$

From (35), we discard the existence of eigenvalues equal to -1 . Recall from Lemma 4.2 that $M_{\mathrm{JOR}}$ has at least $p$ eigenvalues equal to one. Next, we prove that exactly $p$ eigenvalues are equal to 1 , and all the other eigenvalues are strictly smaller than 1 . We will use Lemma C.2 that refer to anchor-based methods, together with relations between eigenvalues of anchor-based and centroid-based systems.

We let $M_{\mathrm{JOR}}^{a}, \Upsilon^{a}, D^{a}, N^{a}$ be as in Def. 4.1. The anchorbased JOR system matrix $M_{\mathrm{JOR}}^{a}$ is related to the anchor-based Jacobi matrix $\left(D^{a}\right)^{-1} N^{a}$ as follows:

$$
\begin{aligned}
& M_{\mathrm{JOR}}^{a}=(1-h) \mathbf{I}_{(n-1) p}+h\left(D^{a}\right)^{-1} N^{a}, \\
& \lambda_{i, r}^{a}\left(M_{\mathrm{JOR}}^{a}\right)=1-h+h \lambda_{i, r}^{a}\left(\left(D^{a}\right)^{-1} N^{a}\right),
\end{aligned}
$$

$\forall i=1, \ldots,(n-1), r=1, \ldots, p$. From Lemma C.2 $\rho\left(\left(D^{a}\right)^{-1} N^{a}\right)<1$. Besides, since $h>0$,

$$
-h<h \lambda_{i, r}^{a}\left(\left(D^{a}\right)^{-1} N^{a}\right)<h .
$$

Using (36) and 37, and following a similar reasoning as in (33)-35), we conclude that, for connected graphs,

$$
-1<1-2 h<\lambda_{i, r}^{a}\left(M_{\mathrm{JOR}}^{a}\right)<1,
$$

$\forall i=1, \ldots,(n-1), r=1, \ldots, p$.

Now, we use a simplified notation for the eigenvalues of matrices $M_{\mathrm{JOR}}^{a}$ and $M_{\mathrm{JOR}}$. We define $\forall r=1, \ldots, p$,

$$
\begin{aligned}
& \lambda_{i, r}=\lambda_{i, r}\left(M_{\mathrm{JOR}}\right), \text { with } i=1, \ldots, n, \text { and } \\
& \lambda_{i, r}^{a}=\lambda_{i, r}^{a}\left(M_{\mathrm{JOR}}^{a}\right), \text { with } i=1, \ldots, n-1 .
\end{aligned}
$$

We consider these eigenvalues are sorted as follows:

$$
\begin{aligned}
& \lambda_{1,1} \leq \ldots \lambda_{1, p} \leq \cdots \leq \lambda_{n, 1} \leq \cdots \leq \lambda_{n, p}, \\
& \lambda_{1,1}^{a} \leq \ldots \lambda_{1, p}^{a} \leq \cdots \leq \lambda_{n-1,1}^{a} \leq \cdots \leq \lambda_{n-1, p}^{a} .
\end{aligned}
$$

From 35, 38, for connected graphs:

$$
\begin{aligned}
& -1<\lambda_{1,1} \leq \cdots \leq \cdots \leq \lambda_{n-1, p} \leq 1, \text { and } \\
& \quad \lambda_{n, 1}=\cdots=\lambda_{n, p}=1, \\
& -1<\lambda_{1,1}^{a} \leq \ldots \lambda_{n-1, p}^{a}<1 .
\end{aligned}
$$

Now we relate the eigenvalues $\lambda_{n-1, p}$ and $\lambda_{n-1, p}^{a}$ to prove that $\lambda_{n-1, p}<1$ for connected graphs. We apply results of symmetric matrices. Although matrices $M_{\mathrm{JOR}}$ and $M_{\mathrm{JOR}}^{a}$ in (12) and Definition 4.1 are not symmetric, from Lemma C.1 they are similar symmetric matrices, and they have the same eigenvalues as their symmetric counterparts. Thus, the eigenvalues of $M_{\mathrm{JOR}}$ and $M_{\mathrm{JOR}}^{a}$ are real.

We can now use [33. Theorem 4.3.15], which applies to Hermitian matrices, using our non-symmetric matrices $M_{\text {JOR }}$ and $M_{\mathrm{JOR}}^{a}$, which states

$$
\lambda_{i, r} \leq \lambda_{i, r}^{a} \leq \lambda_{i+1, r},
$$

$\forall i=1, \ldots,(n-1)$, and $r=1, \ldots, p$. In particular, for $i=$ $n-1$ and $r=p$,

$$
\lambda_{n-1, p} \leq \lambda_{n-1, p}^{a} \leq \lambda_{n, p} .
$$

Since, for connected graphs $\lambda_{n-1, p}^{a}<1$,41, then

$$
\lambda_{n-1, p}<1,
$$

which, together with 35 gives

$$
-1<\lambda_{1,1} \leq \cdots \leq \lambda_{n-1, p}<1,
$$

i.e., the remaining eigenvalues of $M_{\mathrm{JOR}}$ have modulus strictly smaller than 1 , concluding the proof.

\section{D: Proof of Proposition 4.2}

Proof of Proposition 4.2. First, note that $M_{\mathrm{JOR}}$ is diagonalizable by applying Lemma C.1. Hence, considering w the weighting matrix given in Def. 3.1, we can write the left and right eigenvectors of $M_{\mathrm{JOR}}\left(V_{L}\right.$ and $V_{R}$ respectively) as

$$
\begin{aligned}
V_{L}^{T} & =\left[\begin{array}{c}
\mathbf{w}^{T} \\
V_{L_{n-1, p}}^{T} \\
\vdots \\
V_{L_{1,1}}^{T}
\end{array}\right]=\left[\begin{array}{c}
\mathbf{w}^{T} \\
\bar{V}_{L}^{T}
\end{array}\right], \\
V_{R} & =\left[\begin{array}{llll}
\left(\mathbf{1}_{n} \otimes \mathbf{I}_{p}\right) & V_{R_{n-1, p}} & \ldots & V_{R_{1,1}}
\end{array}\right] \\
& =\left[\begin{array}{lll}
\left(\mathbf{1}_{n} \otimes \mathbf{I}_{p}\right) & \bar{V}_{R}
\end{array}\right] .
\end{aligned}
$$

From Lemma 4.2 and eq. (18), w and $\left(\mathbf{1}_{n} \otimes \mathbf{I}_{p}\right)$ are left and right eigenvectors of $M_{\mathrm{JOR}}$ associated to the eigenvalue 1 . $V_{L_{1,1}}, V_{L_{1, p}} \ldots, V_{L_{n-1, p}}$ have been chosen to be orthogonal to $\mathbf{w}$, and $V_{R_{1,1}}, V_{R_{1, p}} \ldots, V_{R_{n-1, p}}$ are orthogonal to $\left(\mathbf{1}_{n} \otimes \mathbf{I}_{p}\right)$. Note that $\left[V_{n, 1}, \ldots, V_{n, p}\right]=\mathbf{w}$ are not necessarily orthogonal among them. 
Let $\lambda_{M}$ be the diagonal matrix with the eigenvalues of $M_{\mathrm{JOR}}$. Then,

$$
\begin{aligned}
V_{L}^{T} M_{\mathrm{JOR}} & =\lambda_{M} V_{L}^{T}, \quad \text { and } M_{\mathrm{JOR}} V_{R}=V_{R} \lambda_{M}, \\
\left(M_{\mathrm{JOR}}\right)^{k} & =V_{R} \lambda_{M}^{k} V_{R}^{-1}=\left(V_{L}^{T}\right)^{-1} \lambda_{M}^{k} V_{L}^{T}, \\
\left(M_{\mathrm{JOR}}\right)^{2 k} & =V_{R} \lambda_{M}^{k}\left(V_{L}^{T} V_{R}\right)^{-1} \lambda_{M}^{k} V_{L}^{T} .
\end{aligned}
$$

We focus on term $\left(V_{L}^{T} V_{R}\right)^{-1}$. From [33, Theorem 1.4.7],

$$
V_{L_{i, r}}^{T} V_{R_{j, s}}=0, \text { for all } \lambda_{i, r}\left(M_{\mathrm{JOR}}\right) \neq \lambda_{j, s}\left(M_{\mathrm{JOR}}\right),
$$

and thus

$$
\begin{aligned}
& V_{L}^{T} V_{R}=\left[\begin{array}{cc}
\mathbf{w}^{T}\left(\mathbf{1}_{n} \otimes \mathbf{I}_{p}\right) & \mathbf{0} \\
\mathbf{0} & \bar{V}_{L}^{T} \bar{V}_{R}
\end{array}\right], \text { and } \\
& \left(V_{L}^{T} V_{R}\right)^{-1}=\left[\begin{array}{cc}
\left(\mathbf{w}^{T}\left(\mathbf{1}_{n} \otimes \mathbf{I}_{p}\right)\right)^{-1} & \mathbf{0} \\
\mathbf{0} & \left(\bar{V}_{L}^{T} \bar{V}_{R}\right)^{-1}
\end{array}\right] .
\end{aligned}
$$

Now we consider $\lambda_{M}$. From Prop. 4.1, for a connected graph,

$$
\begin{aligned}
& \lambda_{n, 1}\left(M_{\mathrm{JOR}}\right)=\cdots=\lambda_{n, p}\left(M_{\mathrm{JOR}}\right)=1, \\
& -1<\lambda_{1,1}\left(M_{\mathrm{JOR}}\right) \leq \cdots \leq \lambda_{n-1, p}\left(M_{\mathrm{JOR}}\right)<1,
\end{aligned}
$$

Thus, $\lambda_{M}=\operatorname{diag}\left(\lambda_{n, p}\left(M_{\mathrm{JOR}}\right), \ldots, \lambda_{1,1}\left(M_{\mathrm{JOR}}\right)\right)$, satisfies:

$$
\lim _{k \rightarrow \infty} \lambda_{M}^{k}=\left[\begin{array}{cc}
\mathbf{I}_{p} & \mathbf{0} \\
\mathbf{0} & \mathbf{0}
\end{array}\right] .
$$

Therefore, using 48,

$$
\begin{aligned}
\lim _{k \rightarrow \infty} & \left(M_{\mathrm{JOR}}\right)^{k}=\lim _{k \rightarrow \infty}\left(M_{\mathrm{JOR}}\right)^{2 k} \\
& =\lim _{k \rightarrow \infty} V_{R} \lambda_{M}^{k}\left(V_{L}^{T} V_{R}\right)^{-1} \lambda_{M}^{k} V_{L}^{T} \\
& =V_{R}\left[\begin{array}{cc}
\mathbf{I}_{p} & \mathbf{0} \\
\mathbf{0} & \mathbf{0}
\end{array}\right]\left(V_{L}^{T} V_{R}\right)^{-1}\left[\begin{array}{cc}
\mathbf{I}_{p} & \mathbf{0} \\
\mathbf{0} & \mathbf{0}
\end{array}\right] V_{L}^{T} \\
& =\left[\left(\mathbf{1}_{n} \otimes \mathbf{I}_{p}\right), \mathbf{0}\right]\left(V_{L}^{T} V_{R}\right)^{-1}\left[\begin{array}{c}
\mathbf{w}^{T} \\
\mathbf{0}
\end{array}\right] \\
& =\left(\mathbf{1}_{n} \otimes \mathbf{I}_{p}\right)\left(\mathbf{w}^{T}\left(\mathbf{1}_{n} \otimes \mathbf{I}_{p}\right)\right)^{-1} \mathbf{w}^{T},
\end{aligned}
$$

as $M_{\star}^{c}$ in Definition 3.1, giving (19).

\section{E: Proof of THEOREM 4.1}

Proof of Theorem 4.1. We let $\mathbf{e}(k)$ be he error containing the difference between the agents estimates $\mathbf{x}(k)$ and the solution $\mathrm{x}^{\star}$ as in Lemma 4.1

$$
\mathbf{e}(k)=\mathbf{x}(k)-\mathbf{x}^{\star}, \quad \mathbf{x}(k)=\mathbf{e}(k)+\mathbf{x}^{\star},
$$

Then, (11)-(12) becomes

$$
\mathbf{e}(k+1)=M_{\mathrm{JOR}} \mathbf{e}(k), \mathbf{e}(k)=\left(M_{\mathrm{JOR}}\right)^{k} \mathbf{e}(0) .
$$

Using Proposition 4.2 .

$$
\lim _{k \rightarrow \infty} \mathbf{e}(k)=\lim _{k \rightarrow \infty}\left(M_{\mathrm{JOR}}\right)^{k} \mathbf{e}(0)=M_{\star}^{c} \mathbf{e}(0) .
$$

Reversing [52] we get

$$
\lim _{k \rightarrow \infty} \mathbf{x}(k)=M_{\star}^{c} \mathbf{x}(0)+\Pi \mathbf{x}^{\star},
$$

with $\Pi$ as in Definition 3.1 and $\mathrm{x}^{\star}$ as in (14). Using Lemma $4.1, \mathbf{x}^{\star}=\hat{\mathbf{x}}_{\mathcal{V}}^{a}+\left(\mathbf{1} \otimes \mathbf{I}_{p}\right) \mathbf{x}_{a}^{\star}$,

$$
\lim _{k \rightarrow \infty} \mathbf{x}(k)=M_{\star}^{c} \mathbf{x}(0)+\Pi \hat{\mathbf{x}}_{\mathcal{V}}^{a}+\Pi\left(\mathbf{1} \otimes \mathbf{I}_{p}\right) \mathbf{x}_{a}^{\star} .
$$

Since $\Pi\left(\mathbf{1} \otimes \mathbf{I}_{p}\right)=\mathbf{0}_{p \times p}$, and $\hat{\mathbf{x}}_{\mathcal{V}}^{c}=\Pi \hat{\mathbf{x}}_{\mathcal{V}}^{a}$ (Definition 3.1, we get 20 .

\section{F: Proofs of Lemmas 5.1 and 6.1}

Proof of Lemma 5.1. The result follows from the proof of Proposition 4.1, using $h<1 / 2$ in (34) to get $0<\lambda_{i, r}\left(M_{\mathrm{JOR}}\right) \leq 1$, instead of 335 .

Proof of Lemma 6.1. From Lemma 5.1. and Prop. 4.1 and 4.2, the eigenvalues of $M_{\mathrm{JOR}}$ are positive, and

$$
\rho_{\text {ess }}\left(M_{\mathrm{JOR}}\right)=\rho\left(M_{\mathrm{JOR}}-M_{\star}^{c}\right)=\lambda_{n-1, p},
$$

with $\lambda_{n-1, p}$ as in (43)-(45). From (38) with $0<h<1 / 2$, the eigenvalues of $M_{\mathrm{JOR}}^{a}$ are positive,

$$
\text { and } \quad \rho\left(M_{\mathrm{JOR}}^{a}\right)=\lambda_{n-1, p}^{a},
$$

with $\lambda_{n-1, p}^{a}$ as in (43). And thus, 21) can be concluded from (43), (57) and (58).

\section{REFERENCES}

[1] A. Petitti, A. Franchi, D. D. Paola, and A. Rizzo, "Decentralized motion control for cooperative manipulation with a team of networked mobile manipulators," in IEEE Int. Conf. on Robotics and Automation, Stockholm, Sweden, May 2016, pp. 441-446.

[2] N. Trawny, X. S. Zhou, K. X. Zhou, and S. I. Roumeliotis, "Inter-robot transformations in 3-d," IEEE Transactions on Robotics, vol. 26, no. 2, pp. 226-243, 2010.

[3] P. Barooah and J. Hespanha, "Distributed estimation from relative measurements in sensor networks," in Int. Conf. on Intelligent Sensing and Information Processing, Chennai, India, Jan. 2005, pp. 88-93.

[4] R. Aragues, L. Carlone, C. Sagues, and G. Calafiore, "Distributed centroid estimation from noisy relative measurements," Systems \& Control Letters, vol. 61, pp. 773-779, 2012.

[5] N. M. Freris and A. Zouzias, "Fast distributed smoothing of relative measurements," in IEEE Conf. on Decision and Control, Maui, Hawaii, Dec. 2012, pp. 1411-1416.

[6] N. Atanasov, R. Tron, V. M. Preciado, and G. J. Pappas, "Joint estimation and localization in sensor networks," in IEEE Conf. on Decision and Control, Los Angeles, CA, Dec. 2014, pp. 6875-6882.

[7] A. Carron, M. Todescato, R. Carli, and L. Schenato, "An asynchronous consensus-based algorithm for estimation from noisy relative measurements," IEEE Transactions on Control of Network Systems, vol. 1, no. 3, pp. 283-295, 2014.

[8] M. Todescato, A. Carron, R. Carli, and L. Schenato, "Distributed localization from relative noisy measurements: a robust gradient based approach," in European Control Conference, Linz, Austria, Jul. 2015, pp. 1914-1919.

[9] M. Todescato, A. Carron, R. Carli, A. Franchi, and L. Schenato, "Multirobot localization via gps and relative measurements in the presence of asynchronous and lossy communication," in European Control Conference, Alborg, Denmark, Jul. 2016, pp. 2527-2532.

[10] C. Ravazzi, P. Frasca, H. Ishii, and R. Tempo, "A distributed randomized algorithm for relative localization in sensor networks," in European Control Conference, Zurich, Switzerland, Jul. 2013, pp. 1776-1781.

[11] W. S. Rossi, P. Frasca, and F. Fagnani, "Limited benefit of cooperation in distributed relative localization," in IEEE Conf. on Decision and Control, Florence, Italy, Dec. 2013, pp. 5427-5431.

[12] C. Ravazzi, N. P. K. Chan, and P. Frasca, "Distributed estimation from relative measurements of heterogeneous and uncertain quality," IEEE Transactions on Signal and Information Processing over Networks, vol. 5, no. 2, pp. 203-217, 2019.

[13] W. S. Rossi, P. Frasca, and F. Fagnani, "Distributed estimation from relative and absolute measurements," IEEE Transactions on Automatic Control, vol. 62, no. 12, pp. 6385-6391, 2017.

[14] _ , "Transient and limit performance of distributed relative localization," in IEEE Conf. on Decision and Control, Maui, Hawaii, 2012, pp. 2744-2748.

[15] D. P. Bertsekas and J. N. Tsitsiklis, Parallel and Distributed Computation: Numerical Methods. Athena Scientific, 1997.

[16] J. Cortes, "Global and robust formation-shape stabilization of relative sensing networks," Automatica, vol. 45, no. 12, pp. 2754-2762, 2009.

[17] S. Choudhary, L. Carlone, C. Nieto, J. Rogers, H. I. Christensen, and F. Dellaert, "Distributed mapping with privacy and communication constraints: Lightweight algorithms and object-based models," Int. Journal of Robotics Research, vol. 36, no. 12, pp. 1286-1311, 2017. 
[18] T. Cieslewski, S. Choudhary, and D. Scaramuzza, "Data-efficient decentralized visual slam," in IEEE Int. Conf. on Robotics and Automation, Brisbane, Australia, May 2018, pp. 2466-2473.

[19] P. Barooah and J. Hespanha, "Error scaling laws for linear optimal estimation from relative measurements," IEEE Transactions on Information Theory, vol. 55, no. 12, pp. 5661-5673, 2009.

[20] D. Seborg, T. Edgar, and D. Mellichamp, Process Dynamics and Control, 1st ED. Chapter 26: Design of Digital Controllers. Wiley, 1989.

[21] J. Wu, V. Ugrinovskii, and F. Allgower, "Cooperative estimation and robust synchronization of heterogeneous multiagent systems with coupled measurements," IEEE Transactions on Control of Network Systems, vol. 5, no. 4, pp. 1597-1607, Dec 2018.

[22] S. S. Kia, J. Hechtbauer, D. Gogokhiya, and S. Martínez, "Serverassisted distributed cooperative localization over unreliable communication links," IEEE Transactions on Robotics, vol. 34, no. 5, pp. 1392 1399, 2018.

[23] C. Wan, G. Jing, S. You, and R. Dai, "Sensor network localization via alternating rank minimization algorithms," IEEE Transactions on Control of Network Systems, 2019.

[24] M. Wei, R. Aragues, C. Sagues, and G. C. Calafiore, "Noisy range network localization based on distributed multidimensional scaling," IEEE Sensors Journal, vol. 15, no. 3, pp. 1872-1883, 2015.

[25] X. Li, X. Luo, and S. Zhao, "Globally convergent distributed network localization using locally measured bearings," IEEE Transactions on Control of Network Systems, 2019.

[26] J. Wang, P. Urriza, Y. Han, and D. Cabric, "Weighted centroid localization algorithm: Theoretical analysis and distributed implementation," IEEE Transactions on Wireless Communications, vol. 10, no. 10, pp. 3403-3413, 2011.

[27] C. Lopez-Limon, M. Franceschelli, C. Seatzu, and A. Ramirez-Trevino, "A consensus algorithm for common reference frame estimation in networked multi-agent systems," in IEEE Conf. on Decision and Control, Los Angeles, CA, Dec. 2014, pp. 6117-6122.

[28] A. Sarlette, R. Sepulchre, and N. E. Leonard, "Autonomous rigid body attitude synchronization," Automatica, vol. 45, no. 2, pp. 572-577, 2008.

[29] B. Lee, S. Kang, and H. Ahn, "Distributed orientation estimation in so(d) and applications to formation control and network localization," IEEE Transactions on Control of Network Systems, 2019.

[30] P. Stegagno and C. Yuan, "Distributed cooperative adaptive state estimation and system identification for multi-agent systems," IET Control Theory \& Applications, vol. 13, no. 6, pp. 815-822, 2019.

[31] R. Olfati-Saber, J. A. Fax, and R. M. Murray, "Consensus and cooperation in networked multi-agent systems," Proceedings of the IEEE, vol. 95, no. 1, pp. 215-233, 2007.

[32] S. S. Kia, B. Van Scoy, J. Cortes, R. A. Freeman, K. M. Lynch, and S. Martinez, "Tutorial on dynamic average consensus: The problem, its applications, and the algorithms," IEEE Control Systems Magazine, vol. 39, no. 3, pp. 40-72, 2019.

[33] R. A. Horn and C. R. Johnson, Matrix Analysis. Cambridge University Press New York, NY, USA, 1986.

[34] L. Elsner and V. Mehrmann, "Convergence of block iterative methods for linear systems arising in the numerical solution of euler equations," Numerische Mathematik, vol. 59, no. 1, pp. 541-559, 1991.

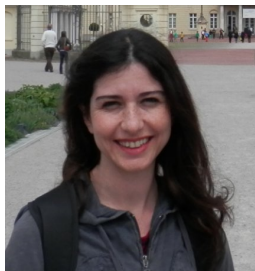

Rosario Aragues received the M.S. and Ph.D. degrees in System Engineering and Computer Science from Univ. Zaragoza, Spain, in 2008 and 2012. She was a postdoctoral researcher at the Institut Pascal, CNRS, UMR 6602, Clermont-Ferrand, France, from April 2012 to October 2013. Currently she works as an Assistant Professor with the Department of Computer Science and Systems Engineering at the University of Zaragoza. Her research interests include multi-robot perception and control using distributed and decentralized strategies.

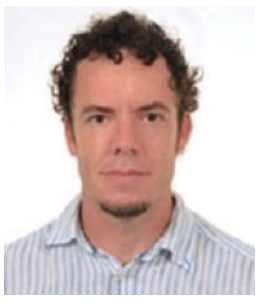

Antonio González received the Telecommunications Engineer degree in 2001 and his Ph.D. in Automation and Industrial Informatics from Universidad Politecnica de Valencia (UPV) in 2012. Currently he works as an Assistant Professor with the Department of Computer Science and System Engineering at the Universidad de Zaragoza (Spain). His research interests are within the broad area of time delay systems, nonlinear control, networked control systems, multirobot systems and process control applications.

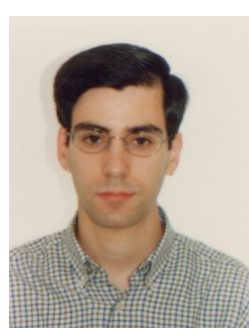

to robotics.
Gonzalo López-Nicolás received the Ph.D. degree in Systems Engineering and Computer Science from the University of Zaragoza, Spain, in 2008. He is Associate Professor with the Department of Computer Science and Systems Engineering at the University of Zaragoza. He is member of the Robotics, Perception and Real-Time Group (RoPerT), and the Aragon Institute of Engineering Research (I3A). His current research interests are focused on visual control, autonomous robot navigation, multirobot systems, and the application of computer vision techniques

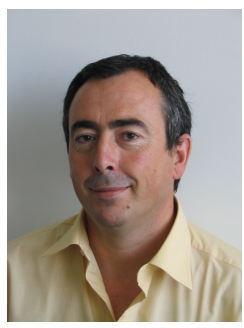

Carlos Sagues received the M.S. degree in computer science and systems engineering and the Ph.D. degree in industrial from the Universidad de Zaragoza, Spain. During the course of his Ph.D. he worked on force and infrared sensors for robotics. In 1994 he became an Associate Professor with the Departamento de Informática e Ingeniería de Sistemas, Universidad de Zaragoza, where he has also occupied the Head Teacher position. Since 2009, he has been a Full Professor with this department. He is a member of the Instituto de Investigación en Ingeniería de Aragón. His current research interests include control systems, computer vision, visual robot navigation, and multivehicle cooperative control. 